\title{
Recent progress in String Field Theory
}

\section{L*.Bonora}

International School for Advanced Studies, Via Beirut 2-4, 34014 Trieste, Italy, and INFN, Sezione di Trieste

E-mail: bonoraesissa.it

This is short review of tachyon condensation and open string field theory. After a brief introduction to open string theory, the SFT action is introduced and illustrated. Next comes tachyon condensation in the level truncation approach, which introduces the main topic: the description of the analytic solution and the proof of the first two conjectures by Sen. The third conjecture is discussed in the framework of vacuum SFT. Finally the subject of open-closed string duality is tackled by commenting about an attempt at showing a more explicit connection between open and closed strings.

5th International School on Field Theory and Gravitation,

April 20 - 242009

CuiabÃa city, Brazil

\footnotetext{
${ }^{*}$ Speaker.
} 


\section{Introduction}

Tachyon condensation is a pervasive phenomenon in physics. Whenever a field theory has a potential with a local maximum, surrounded by (possibly a continuum of) local minima, quantization around the maximum brings about the appearance of an unphysical particle with negative square mass, the tachyon. The tachyon is simply the manifestation of the instability of the vacuum chosen to quantize the theory. Any tiny disturbance takes the system to a more stable configuration based on a local minimum (the tachyons have condensed). This is, for instance, the typical situation of the spontaneous breakdown of a symmetry. The subject of these lectures is tachyon condensation in a system of infinite many particles, as described by string field theory (SFT). The motivations underlying the study of this system are both theoretical and applicative, and stem from the overwhelming role D-branes have assumed in the description of physical systems in the framework of string theory.

D-branes mean open strings: open strings (unlike closed strings) do not exist as autonomous entities but only when their endpoints can lie on D-branes (which, as the case may be, may fill the space). On the other hand D-branes do not have an autonomous existence either: they are a geometrical abstraction representing the dynamics of the open strings attached to them. Studying the dynamics of open strings is therefore of upmost importance and tachyon condensation is basic in this respect. An example may be more illuminating than many words. A phenomenon like inflation can be described by the attractive potential between a D-brane and an anti-D-brane, at least as long as the two branes are far apart. However, when their distance becomes smaller than the string scale (after inflation has terminated) the string spectrum develops tachyons and the natural evolution of the system is represented by tachyon condensation.

In these lectures I will discuss bosonic open string field theory. Purely bosonic string theory is, of course, by itself insufficient, if anything because its spectrum does not contain fermions. However open string field theory is a simplified playground with respect to the corresponding superstring field theory versions. Exploiting the relative simplicity of the bosonic theory it has been possible in the last ten years to make significant progress and, then, export it to some extent to the superstring relatives. Therefore our playground will be the description of tachyon condensation and related phenomena in the framework of Witten's Open String Field Theory (Witten 1987), and the guideline for all these recent developments is represented by A.Sen's conjectures (Sen 1998, 1999). The latter can be summarized as follows. Bosonic open string theory in $D=26$ dimensions is quantized on an unstable vacuum, an instability which manifests itself through the appearance of the open string tachyon. The effective tachyonic potential has, beside the local maximum where the theory is quantized, a local minimum. Sen's conjectures concern the nature of the theory around this local minimum. First of all, the energy density difference between the maximum and the minimum should exactly compensate for the D25-brane tension characterizing the unstable vacuum (first conjecture): this is a condition for the (relative) stability of the theory at the minimum. Therefore the theory around the minimum should not contain any quantum fluctuation pertaining to the original (unstable) open string theory (second conjecture). The minimum should therefore correspond to an entirely new theory, which can only be the bosonic closed string theory. If so, in the new theory one should be able to find in particular all the classical solutions characteristic of closed string theory, the D25-brane as well as all the solitonic solutions representing lower 
dimensional D-branes (third conjecture).

The evidence in favor of these conjectures has accumulated over the years although not with a uniform degree of accuracy and reliability, until the first two conjectures were rigorously proved (Schnabl 2006, Ellwood and Schnabl 2007): an explicit analytic (non-perturbative) SFT solution was provided which links the initial vacuum to the final one and it was shown that this vacuum does not contain perturbative open string modes. As for the third conjecture the most important evidence we have gathered so far of solitonic solutions comes from the Vacuum String Field Theory (VSFT), an approximate version of the full SFT, which is believed to represent rather faithfully the theory near the minimum, at least as far as static solutions are concerned.

The D25-brane and its lower dimensional companions are unstable, because there is no conserved charge (like in the corresponding supersymmetric theories) associated to them. Therefore SFT must contain also time-dependent solutions that describe their decay. This issue has been discussed (Sen 2002, 2003a) and approximate solutions have been found in SFT, but exact solutions are still lacking.

Finally, a very far-reaching consequence of Sen's conjectures is so far remained rather implicit in the literature. It is evident that if the three conjectures are true and the new vacuum is the closed string vacuum, then it means that the closed string degrees of freedom can be represented (although non-perturbatively) in terms of the open string ones. This is an exciting possibility that has not been methodically explored so far.

The aim of this review is not a full account of the entire subject of SFT and tachyon condensation, which would take an article the size of a book. There are already several reviews the reader can consult (Ohmori 2001, Are'feva et al. 2002, Bonora et al 2003, Taylor and Zwiebach 2003, Fuchs and Kroyter 2008), which cover different aspects and different subjects. My aim is to give a general survey and convey the main messages without insisting too much, wherever possible, on too many details.

\section{Open string field theory}

Before we come to the formal definition of string field theory, i.e. second quantized string theory, we need a short summary of first quantized open string theory.

\subsection{First quantized open strings}

First quantized open string theory in the critical dimension $\mathrm{D}=26$ is formulated in terms of quantum oscillators $\alpha_{n}^{\mu},-\infty<n<\infty, \mu=0,1, \ldots, 25$, which come from the mode expansion of the string scalar field

$$
X^{\mu}(z)=\frac{1}{2} x^{\mu}-\frac{i}{2} p^{\mu} \ln z+\frac{i}{\sqrt{2}} \sum_{n \neq 0} \frac{\alpha_{n}^{\mu}}{n} z^{-n}
$$

having set the characteristic square length of the string $\alpha^{\prime}=1$. They satisfy the algebra $\left[\alpha_{m}^{\mu}, \alpha_{n}^{\nu}\right]=$ $m \eta^{\mu v} \delta_{n+m, 0}, \eta$ being the space-time Minkowski metric. The vacuum is defined by $\alpha_{n}^{\mu}|0\rangle=0$ for $n>0$ and $p^{\mu}|0\rangle=0$. The states of the theory are constructed by applying to the vacuum the remaining quantum oscillators $\alpha_{n}^{\mu^{\dagger}}=\alpha_{-n}^{\mu}$, with $n>0$. Any such state $|\phi\rangle$ is given momentum $k^{\mu}$ 
by multiplying it by the eigenstate $e^{i k x}$. This state with momentum will be denoted by $|\phi, k\rangle$. In order for such states to be physical they must satisfy the conditions

$$
L_{n}^{(X)}|\phi, k\rangle=0, \quad n>0, \quad\left(L_{0}^{(X)}-1\right)|\phi, k\rangle=0
$$

where $L_{n}^{(X)}$ are the matter Virasoro generators

$$
L_{n}^{(X)}=\frac{1}{2}: \sum_{k=-\infty}^{\infty} \alpha_{n-k}^{\mu} \alpha_{k}^{v}: \eta_{\mu v}
$$

where we have set $\alpha_{0}=p$ and :: denotes normal ordering. The conditions (2.1) are the quantum translation of the classical vanishing of the energy-momentum tensor.

The conditions (2.1) define the physical spectrum of the theory (in $\mathrm{D}=26$ ). All the states are ordered according to the level, the level being a natural number specified by the eigenvalue of $L_{0}^{(X)}+L_{0}^{(g h)}-p^{2}$. The lowest lying state (level 0 ) is the tachyon represented by the vacuum with momentum $k$ and square mass $M^{2}=-1$. The next (level 1) is the massless vector state $\zeta_{\mu} \alpha_{-1}^{\mu}|0\rangle e^{i k x}$ with $k^{2}=0$ and $\zeta \cdot k=0$, which is interpreted as a gauge field. The other states are all massive, with increasing masses proportional to the Planck mass square.

To each of these states is associated a vertex operator. For instance, to the tachyon we associate $V_{t}(k)=: e^{i k \cdot X}: ;$ to the vector state $V_{A}(k, \zeta)=: \zeta \cdot \dot{X} e^{i k \cdot X}:$, where the dot on top of $X$ denotes the tangent derivative with respect to the world-sheet boundary (the real axis in the $z$ UHP); and so on. In this way one can formulate rules to calculate any kind of amplitude of these operators $\left\langle V_{1}\left(k_{1}\right) \ldots V_{N}\left(k_{N}\right)\right\rangle$, as far as these amplitudes are on shell. At low energy $\alpha^{\prime} \rightarrow 0$ such amplitudes reproduce those of the corresponding field theory (for instance, the amplitudes of $V_{A}$ reproduce the amplitudes of a Maxwell field theory). If we want to compute off-shell amplitudes, in general we have to resort to a field theory of strings. This was one of the original motivations for introducing a string field theory.

So far we have ignored ghosts. Indeed the $b, c$ ghosts, which come from the gauge fixing of reparametrization invariance via the Faddeev-Popov recipe, play a minor role in perturbative string theory. They play a much more important role in SFT. They are also expanded in modes $c_{n}$ and $b_{n}$ and one can construct the corresponding Virasoro generators

$$
L_{n}^{(g h)}=: \sum_{k}(2 n+k) b_{-k} c_{k+n}:
$$

Both (2.2) and (2.3) obey the same Virasoro algebra

$$
\left[L_{n}, L_{m}\right]=(n-m) L_{n+m}+\frac{c}{12}\left(n^{3}-n\right)
$$

The central charge $c$ equals the number of $X$ fields in the matter case (i.e. 26), while it equals -26 in the case of the $b, c$ ghosts. So the total central charge vanishes in $\mathrm{D}=26$. This guarantees the absence of any trace anomaly, and therefore consistency of the bosonic string theory as a gauge theory.

The previous results about ghosts and critical dimension, can be usefully reformulated in terms of BRST symmetry and its charge $Q . Q$ is defined by

$$
Q=\sum_{n}: c_{n}\left(L_{n}^{(X)}+\frac{1}{2} L_{n}^{(g h)}\right):
$$


It is hermitean $Q^{\dagger}=Q$ and its basic property is nilpotency

$$
Q^{2}=0
$$

in critical dimension. The study of the physical spectrum can be reformulated in terms of the cohomology of $Q$ : the physical states of perturbative string theory are the states of ghost number 1 that are annihilated by $Q$, defined up to states obtained by acting with $Q$ on any state of ghost number 0 . They can be represented by the old physical states $|\phi, k\rangle$ tensored with the ghost factor $c_{1}|0\rangle$.

With this at hand we can now turn to string field theory.

\subsection{The SFT action and star product}

The open string field theory action proposed by E.Witten years ago (Witten 1987) is

$$
\mathscr{S}(\Psi)=-\frac{1}{g_{o}^{2}} \int\left(\frac{1}{2} \Psi * Q \Psi+\frac{1}{3} \Psi * \Psi * \Psi\right) .
$$

This action is clearly reminiscent of the Chern-Simons action in 3D. In this expression $\Psi$ is the string field. It can be understood either as a classical functional of the open string configurations $\Psi\left(x^{\mu}(z)\right)$, or as a vector in the Fock space of states of the open string theory. Altough the first representation is more pictorial, the second is far more effective from a practical viewpoint. In the following we will consider for simplicity only this second point of view. In the field theory limit it makes sense to represent $\Psi$ as a superposition of Fock space states with ghost number 1, with coefficient represented by (infinite many) local fields,

$$
|\Psi\rangle=\left(\phi(x)+A_{\mu}(x) a_{1}^{\mu \dagger}+\ldots\right) c_{1}|0\rangle .
$$

The BRST charge $Q$ is the same as the one introduced above for the first quantized string theory.

One of the most fundamental ingredients is the star product. Physically it represents the string interaction, that is the process of two strings coming together to form a third string. More precisely the product of two string fields $\Psi_{1}, \Psi_{2}$ represents the process of identifying the right half of the first string with the left half of the second string and integrating over the overlapping degrees of freedom, to produce a third string which corresponds to $\Psi_{1} * \Psi_{2}$. This can be implemented in different ways, either using the classical string functional (as in the original formulation by Witten), or using the three string vertex (see below), or the conformal field theory language (Leclair et al. 1989).

Finally the integration in (2.6) corresponds to bending the left half of the string over the right half and integrating over the corresponding degrees of freedom in such a way as to produce a number.

The following rules are obeyed

$$
\begin{aligned}
& Q^{2}=0 \\
& \int Q \Psi=0, \\
& \left(\Psi_{1} * \Psi_{2}\right) * \Psi_{3}=\Psi_{1} *\left(\Psi_{2} * \Psi_{3}\right), \\
& Q\left(\Psi_{1} * \Psi_{2}\right)=\left(Q \Psi_{1}\right) * \Psi_{2}+(-1)^{\left|\Psi_{1}\right|} \Psi_{1} *\left(Q \Psi_{2}\right),
\end{aligned}
$$


where $|\Psi|$ is the Grassmannality of the string field $\Psi$, which, for bosonic strings, coincides with the ghost number. The action (2.6) is invariant under the BRST transformation

$$
\delta \Psi=Q \Lambda+\Psi * \Lambda-\Lambda * \Psi .
$$

Finally, the ghost numbers of the various objects $Q, \Psi, \Lambda, *, \int$ are $1,1,0,0,-3$, respectively.

Let us now see in more detail how to implement the star product. Let us consider three unit semi-disks in the upper half $z_{a}(a=1,2,3)$ plane. Each one represents the string freely propagating in semicircles from the origin (world-sheet time $\tau=-\infty$ ) to the unit circle $\left|z_{a}\right|=1(\tau=0)$, where the interaction is supposed to take place. We map each unit semi-disk to a $120^{\circ}$ wedge of the complex plane via the following conformal maps:

$$
f_{a}\left(z_{a}\right)=\alpha^{2-a} f\left(z_{a}\right), a=1,2,3,
$$

where

$$
f(z)=\left(\frac{1+i z}{1-i z}\right)^{\frac{2}{3}}
$$

Here $\alpha=e^{\frac{2 \pi i}{3}}$. In this way the three semi-disks are mapped to non-overlapping (except along the edges) regions in such a way as to fill up a unit disk centered at the origin. The curvature is zero everywhere except at the center of the disk, which represents the common midpoint of the three strings in interaction, see Fig.(1)

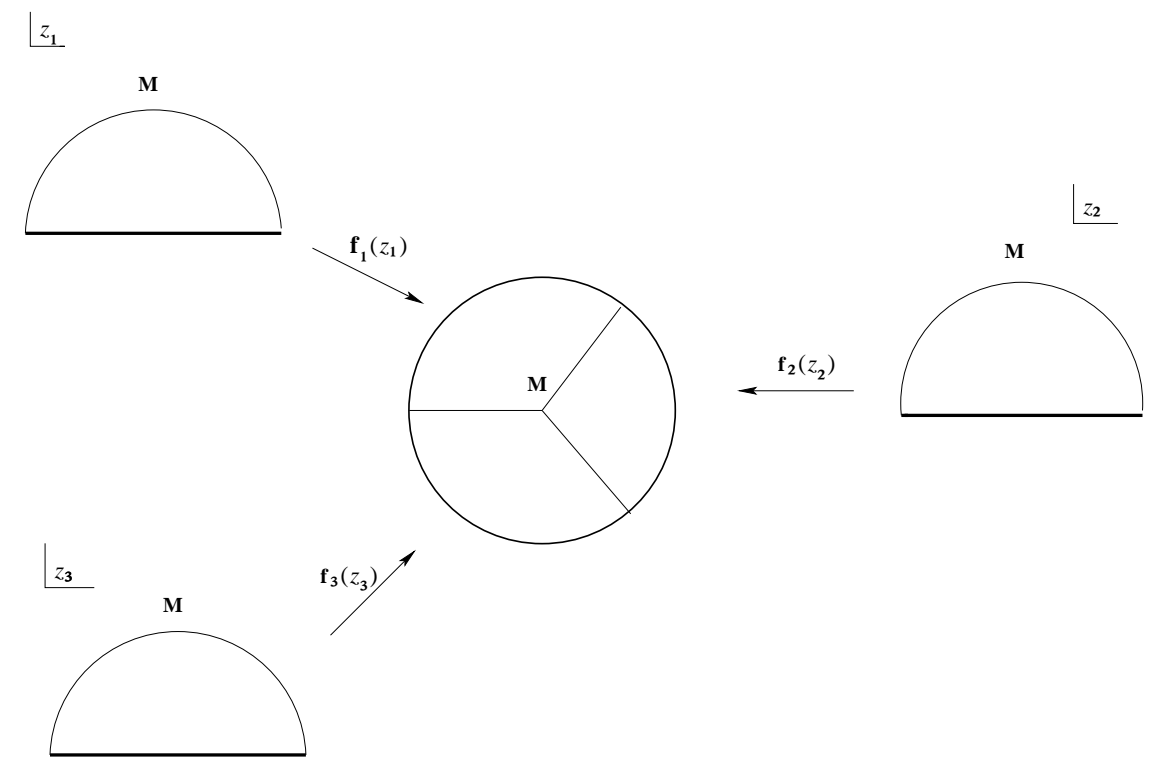

Figure 1: The conformal maps from the three unit semi-disks to the three-wedges unit disk

The interaction vertex is defined by means of a correlation function on the disk in the following way

$$
\int \psi * \phi * \chi=\left\langle f_{1} \circ \psi(0) f_{2} \circ \phi(0) f_{3} \circ \chi(0)\right\rangle
$$

So, calculating the star product amounts to evaluating a three point function on the unit disk. 


\section{Tachyon condensation}

Following the rules of the previous section it is possible to explicitly compute the action (2.6). For instance, in the low energy limit, where the string field may be assumed to take the form (2.7), the action becomes an integrated function $F$ of an infinite series of local polynomials (kinetic and potential terms) of the fields involved in (2.7):

$$
\mathscr{S}(\Psi)=\int d^{26} x F\left(\varphi_{i}, \partial \varphi_{i}, \ldots\right)
$$

To limit the number of terms one has to limit the gigantic BRST symmetry of OSFT, by choosing a gauge, which is usually the Feynman-Siegel gauge: this means that we limit ourselves to the states that satisfy the condition: $b_{0}|\Psi\rangle=0$

Still the action with all the infinite sets of fields contained in $\Psi$ remains unwieldy. As it turns out, it makes sense to limit the number of fields in $\Psi$, provided we insert all the fields up to a certain level. This is called level truncation and turns out to be an excellent approximation and regularization scheme in SFT. Let us see this in more detail for a string field which includes the tachyon $\phi(x)$ and the vector field $A_{\mu}(x)$. The action turns out to be (Ohmori 2001)

$$
\begin{aligned}
\mathscr{S}_{(0,1)}= & \frac{1}{g_{o}^{2}} \int d^{26} x\left(-\frac{1}{2} \partial_{\mu} \phi \partial^{\mu} \phi+\frac{1}{2} \phi^{2}-\frac{1}{3} \beta^{3} \phi^{3}-\frac{1}{2} \partial_{\mu} A_{\nu} \partial^{\mu} A^{v}\right. \\
& \left.-\beta \tilde{\phi} \tilde{A}_{\mu} \tilde{A}^{\mu}-\frac{\beta}{2}\left(\partial_{\mu} \partial_{\nu} \tilde{\phi} \tilde{A}^{\mu} \tilde{A}^{v}+\tilde{\phi} \partial_{\mu} \tilde{A}^{v} \partial_{\nu} \tilde{A}^{\mu}-2 \partial_{\mu} \tilde{\phi} \partial_{\nu} \tilde{A}^{\mu} \tilde{A}^{v}\right)\right)
\end{aligned}
$$

where $\beta=\frac{3 \sqrt{3}}{4}$ is a recurrent number in SFT. One can see the kinetic term for the tachyon and the gauge field (the latter is in the gauge fixed form because the Feynman-Siegel gauge corresponds in the field theory language to the Lorentz gauge) and the 'wrong' mass term for the tachyon. The fields appearing in the interactions terms carry a tilde. This means, for any field $\varphi$

$$
\tilde{\varphi}(x)=e^{-\ln \left(\beta^{-1} \partial_{\mu} \partial^{\mu}\right)} \varphi(x)
$$

Incidentally, the fact that the interaction is formulated in terms of tilded fields is a manifestation of the strong (exponential) convergence properties of string theory in the UV.

Let us now consider the potential and study its minimum. We remind the reader that this theory is supposed to represent the open strings attached to a space-filling D-brane, the D25brane. It may also represent lower dimensional branes. In the CFT language such configurations are described by boundary CFT's. The first important remark (Sen 1998) is that this potential is universal, it does not depend on the details of the theory, i.e. on a particular boundary conformal field theory.

Let us concentrate on the D25-brane and evaluate the total energy of the system brane + string modes. The brane has its intrinsic energy, whose density is the tension $\tau$, which in our conventional units $\left(\alpha^{\prime}=1\right)$, is given by $\tau=\frac{1}{2 \pi^{2} g_{o}^{2}}$. The string modes are represented by the action and, in a static situation, their total energy is given by the negative action. We precisely wish to study this system in the vacuum. Since we want Lorentz invariance, only Lorentz scalars can acquire a VEV. Therefore in (3.2) one must set the tensor fields and all the derivatives to 0 . Setting $\langle\phi\rangle=t$, what remains of 
the action (divided by the total volume) can be written in terms of the function $u(t)$ as follows

$$
-\frac{S}{V} \equiv \tau u(t)=2 \pi^{2}\left(-\frac{1}{2} t^{2}+\frac{1}{3} \beta^{3} t^{3}\right)
$$

This is the total tachyon potential energy density extracted from the action.

The total energy of the system will be given by the sum of (3.3) and the D25-brane tension

$$
U(t)=\tau(1+u(t))
$$

This potential is cubic, and it is easy to determine both local maximum and minimum. The latter is given by

$$
t=t_{0}=\frac{1}{\beta^{3}}, \quad u(t) \approx-0.684
$$

Let us recall that the first conjecture by Sen is that the tachyonic energy should exactly compensate for the D25-brane tension. Therefore (3.5) does not match this result, but we should remember that ours has been a very rough approximation, since we have retained only two fields, the tachyon and the Maxwell field. It can be shown that by adding more and more fields to the string fields $\Psi$, that is truncating it at a higher level, the value of $u\left(t_{0}\right)$ gets closer and closer to -1 . The asymptotic situation is represented in Fig.(2)

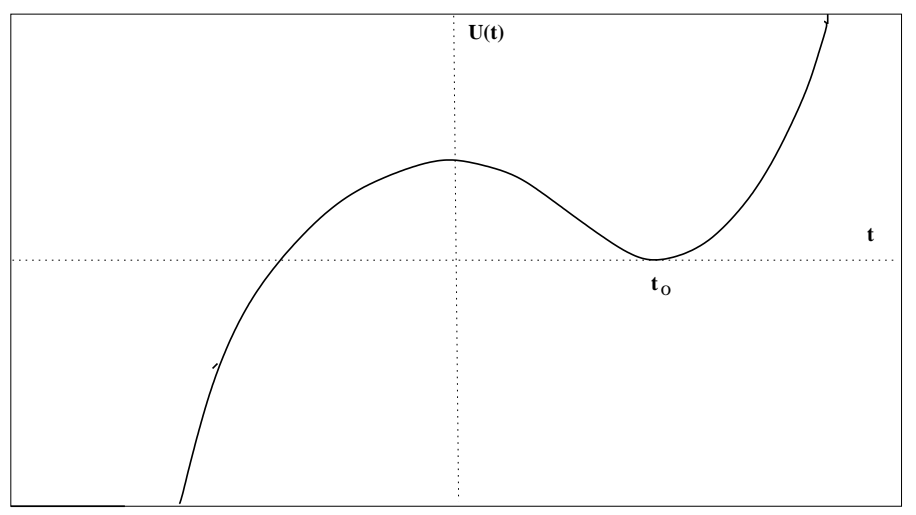

Figure 2: The tachyon potential

This was historically the first evidence that the first Sen's conjecture is correct.

\section{The analytic solution}

In this section I will explain how the first analytic solution to the SFT equation of motion (5.3) was found (Schnabl 2006). This solution is a string state that specifies the (locally) stable vacuum, to be identified as the closed string vacuum. In the oversimplified language of the figure (2) it would correspond to $\left|\Psi_{0}\right\rangle=t_{0} c_{1}|0\rangle$, but it actually identifies the vev of all the infinite many scalar fields that feature in the most general string field.

To start with I have to introduce one of the important ingredients of this solution, the wedge states. 


\subsection{Wedge states and the new coordinate patch}

Wedge states are particular surface states. The latter are states simply defined by a map from the half-disk to the unit disk or, equivalently, to the upper half plane. The definition is as follows: take any map $f$ from the half-disk to a surface $\Sigma$ (inscribed in the unit disk or in the UHP); consider any field $\phi$ and the state $|\phi\rangle=\phi(0)|0\rangle$ in the Fock space of the theory; then the surface state $\langle S|$ is defined by

$$
\langle S \mid \phi\rangle=\langle f \circ \phi\rangle_{\Sigma}
$$

The definition is implicit and may seem at first not very handy, but one can reduce the calculation to very simple test states $|\phi\rangle$, much in the same way as we do in calculating the Neumann coefficients for the three strings vertices in Appendix. One can see that a surface state can be written as a squeezed state represented by a Neumann matrix $S_{n m}$, both for the matter and the ghost part.

Wedges states are particularly simple. Their defining functions are

$$
f_{r}(z)=\left(\frac{1+i z}{1-i z}\right)^{\frac{2}{r}}
$$

where, for simplicity, we take $r$ to be a positive integer. This means that the image of the map is a wedge of angle $\frac{2 \pi}{r}$ in the unit disk. They can be shown to satisfy the recursion relation

$$
|r\rangle \star|s\rangle=|r+s-1\rangle
$$

In particular we see that calling $|\Xi\rangle$ the result of taking $r \rightarrow \infty$ in $|r\rangle$, we recover $\Xi^{2}=\Xi$. This may seem formal, but it can be shown to give rise precisely to the sliver, which is a surface state defined by a wedge of vanishing angle (see next section for a more accurate definition). So, in particular, wedge states approximate the sliver.

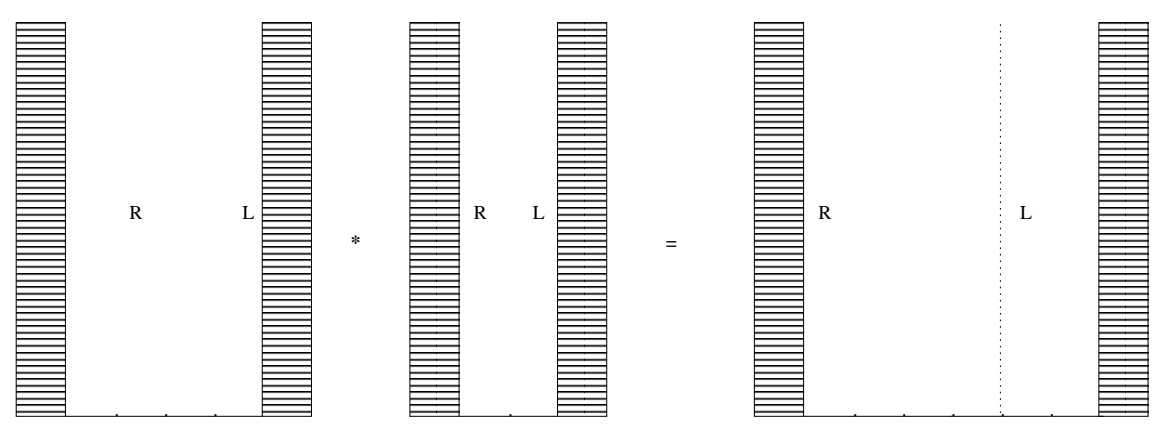

Figure 3: Star product of two wedge states $|3\rangle \star|2\rangle=|4\rangle$

The star product of wedge states takes a particularly simple form if we use the coordinate $\tilde{z}=\arctan z$. In this new representation a wedge state $|r\rangle$ is a cylinder in the $\tilde{z}$ UHP, see fig.(3). It is in fact an infinite strip in the imaginary direction of width $r \frac{\pi}{2}$. It is formed by two external strips of width $\frac{\pi}{4}$ each (the ruled strips in the figure), and an internal strip of width $(r-1) \frac{\pi}{2}$. The rightmost and leftmost sides are identified so as to form a cylinder. The star product of two such states is 
simply obtained by dropping the rightmost ruled strip of the first state and the leftmost ruled strip of the second and gluing the two cut cylinders along the dashed line in fig.(3). In this language the wedge state with $r=2$ corresponds to the vacuum $|0\rangle$.

Pure wedge states, as we have just described them, are not enough to describe the analytic solution we are looking for. We need wedge states with insertion, that is wedge states with the insertion of an operator at some point of the unruled patches. The $|n\rangle$ wedge state itself can be seen as such.

$$
|n\rangle=\left(\frac{2}{n}\right)^{\mathscr{L}_{0}^{\dagger}}|0\rangle
$$

where $\mathscr{L}_{0}$ will be introduced in a moment.

These states will play a major role in what follows. What we need now is exploit the new coordinate $\tilde{z}=\arctan z$ to get a few basic definitions and relations. To start with we define the Virasoro generators in the new coordinate patch

$$
\mathscr{L}_{0}=\oint \frac{d \tilde{z}}{2 \pi i} \tilde{z} T_{\tilde{z} \tilde{z}}(\tilde{z})
$$

that is

$$
\mathscr{L}_{0}=L_{0}+\sum_{k=1}^{\infty} \frac{2(-1)^{k+1}}{4 k^{2}-1} L_{2 k}
$$

as well as $\mathscr{L}_{ \pm 1}$. They satisfy $\left[\mathscr{L}_{n}, \mathscr{L}_{m}\right]=(n-m) \mathscr{L}_{n+m}$.

Other useful operators are

$$
\begin{aligned}
\mathscr{B}_{0} & =b_{0}+\sum_{k=1}^{\infty} \frac{2(-1)^{k+1}}{4 k^{2}-1} b_{2 k} \\
B_{1} & =b_{1}+b_{-1}
\end{aligned}
$$

and

$$
\begin{aligned}
B \equiv B_{1}^{L} & =\frac{1}{2} B_{1}+\frac{1}{\pi}\left(\mathscr{B}_{0}+\mathscr{B}_{0}^{\dagger}\right) \\
B_{1}^{R} & =\frac{1}{2} B_{1}-\frac{1}{\pi}\left(\mathscr{B}_{0}+\mathscr{B}_{0}^{\dagger}\right)
\end{aligned}
$$

and using $K_{1}=L_{1}+L_{-1}$ we can introduce

$$
\begin{aligned}
K \equiv K_{1}^{L} & =\frac{1}{2} K_{1}+\frac{1}{\pi}\left(\mathscr{L}_{0}+\mathscr{L}_{0}^{\dagger}\right) \\
K_{1}^{R} & =\frac{1}{2} K_{1}-\frac{1}{\pi}\left(\mathscr{L}_{0}+\mathscr{L}_{0}^{\dagger}\right)
\end{aligned}
$$

For instance we have the 'semi-derivation' rules

$$
\begin{aligned}
& K_{1}^{L}\left(\Psi_{1} \star \Psi_{2}\right)=\left(K_{1}^{L} \Psi_{1}\right) \star \Psi_{2} \\
& K_{1}^{R}\left(\Psi_{1} \star \Psi_{2}\right)=\Psi_{1} \star\left(K_{1}^{R} \Psi_{2}\right)
\end{aligned}
$$

and the wedge states can also be written as

$$
|n\rangle=e^{\frac{\pi}{2}(n-1) K}|1\rangle
$$

From this equation and (4.4) we see that it makes sense to consider $n$ a real variable rather than an integer, and therefore also to differentiate with respect to it. 


\subsection{The solution}

Schnabl chose the gauge $\mathscr{B}_{0}|\Psi\rangle=0$, rather than the Feynman-Siegel one. He than made the ansatz

$$
\Psi=\lim _{N \rightarrow \infty}\left(\sum_{n=0}^{N} \psi_{n}^{\prime}-\psi_{N}\right)
$$

where

$$
\psi_{n}=c_{1}|0\rangle \star B|n\rangle \star c_{1}|0\rangle
$$

and the prime denotes derivative with respect to $n$. The state $\psi_{n}$ is made out of wedges states with insertions of the field $c$ and of $B$. In particular for $n=0$ we have

$$
\psi_{0}=(c B c)(0)|0\rangle, \quad \psi_{0}^{\prime}=(c B K c)(0)|0\rangle
$$

We remark that in the RHS of (4.6) the second term $-\psi_{N}$ is added only for regularization purposes.

The solution is obtained as a limit and it is constructed as

$$
\Psi_{\lambda}=\sum_{n=0}^{\infty} \lambda^{n+1} \psi_{n}^{\prime}
$$

This is a pure gauge solution (action=0) for $\lambda<1$, but it is not pure gauge anymore for $\lambda=1$ and it is the good solution. We will not prove it here. Rather we concentrate on the evidence about first Sen's conjecture.

\subsection{First and second Sen's conjectures}

From the equation of motion we get

$$
\langle\Psi, Q \Psi\rangle=-\langle\Psi, \Psi \star \Psi\rangle
$$

This equation has to be explicitly checked over the solution (4.6) - a rather nontrivial task -, because one of the subtleties of SFT is that, even if $|\Psi\rangle$ is a solution to the equation of motion, it is not automatically guaranteed that (4.9) holds.

On the other hand, from the explicit form of the solution one gets

$$
\langle\Psi, Q \Psi\rangle=-\frac{3}{\pi^{2}}
$$

Therefore, finally, the total energy of the string modes is ( $V$ is the total 26-th dimensional volume):

$$
E=-\frac{S}{V}=\frac{1}{g_{o}^{2} V}\left(\frac{1}{2}\langle\Psi, Q \Psi\rangle+\frac{1}{3}\langle\Psi, \Psi \star \Psi\rangle\right)=-\frac{1}{2 \pi^{2} g_{0}^{2}}
$$

which is precisely the negative of the D25-brane tension $\tau$.

Let us now pass to briefly illustrate the proof of the second conjecture (Ellwood and Schnabl 2007). The purpose is to show that the cohomology about Schnabl's solution is trivial. Relabeling 
Schnabl's solution as $\Psi_{0}$, we are looking now for solutions to (5.3) of the type $\Psi_{0}+\psi$, linearized on $\psi$. It is easy to see that the relevant (linearized) equation of motion is

$$
\mathscr{Q} \psi \equiv Q \psi+\Psi_{0} \star \psi-(-1)^{|\psi|} \psi \star \Psi_{0}
$$

This defines a new BRST operator $\mathscr{Q}$ (indeed $\mathscr{Q}^{2}=0$ ) and defines the cohomology around Schnabl's solution. The purpose is to prove that this cohomology is empty.

Let us introduce the symbol

$$
W_{r}=|r+1\rangle
$$

Next let us define the state

$$
A=-\frac{2}{\pi} B \int_{0}^{1} W_{r} d r
$$

Once again we make use of the fact that wedge states can be defined for any real label $r$, not just for an integral $r$. It is possible to prove that

$$
\mathscr{Q} A=|1\rangle
$$

where the RHS represents the wedge state with $r=1$. This is the identity state and satisfies

$$
|1\rangle \star \Phi=\Phi \star|1\rangle=\Phi
$$

for any $\Phi$.

Now suppose $\psi$ satisfies $\mathscr{Q} \psi=0$, then, using these results, we get

$$
\mathscr{Q}(A \star \psi)=(\mathscr{Q} A) \star \psi-A \star(\mathscr{Q} \psi)=|1\rangle \star \psi=\psi
$$

which means that $\psi$ is BRST trivial. This is a very general result. It implies not only that the cohomology of ghost number 1 is trivial (i.e., there is no physical perturbative string mode in the new vacuum), but that the cohomology is trivial for any ghost number state.

\section{The third conjecture}

The third of Sen's conjectures has not been proven analytically so far, the reason being that for this purpose one cannot use the elegant and simple analytic methods of the previous section. In fact the third conjecture predicts the existence of lower dimensional solitonic solutions (specifically Dp-branes, with $p<25$ ). But these solutions bring along the breaking of translational symmetry and background dependence. So far the evidence for such solutions is overwhelming, but no exact example has been found yet. It has been possible to find them with approximate methods or with exact methods but in related theories.

A related theory which has brought about significant developments has been the so called vacuum string field theory (VSFT). VSFT, (Rastelli et al. 2001), is a version of Witten's open SFT which is supposed to describe the theory at the minimum of the tachyonic potential. The argument is as follows: let us consider Schnabl's solution and call it $\Psi_{0}$; the generic string field in 
the SFT action can be rewritten by shifting $\Psi \rightarrow \Psi_{0}+\Psi$. It is easy to see that the action maintains the same functional form as the original SFT action. But the form of the BRST charge becomes very complicated (see (4.11) above). One can at this point try to simplify it with the help of some heuristic argument in order to complement our ignorance. Relying on the evidence that, at the minimum, the negative tachyonic potential exactly compensates for the D25-brane tension one can conclude that no open string mode should be excited. So that the BRST cohomology must be trivial. The possible BRST operators that satisfy this condition are of course manifold. However it is possible to find evidence that a consistent form is (5.2) below. This does not mean that VSFT is equivalent to the true theory, but simply that it is a consistent simplification thereof, near the potential minimum ${ }^{1}$.

In short the formulas relevant to VSFT are as follows. The action is

$$
\mathscr{S}(\Psi)=-\frac{1}{g_{0}^{2}}\left(\frac{1}{2}\langle\Psi|\mathscr{Q}| \Psi\rangle+\frac{1}{3}\langle\Psi \mid \Psi * \Psi\rangle\right),
$$

where

$$
\mathscr{Q}=c_{0}+\sum_{n>0}(-1)^{n}\left(c_{2 n}+c_{-2 n}\right)
$$

The equation of motion is

$$
\mathscr{Q} \Psi=-\Psi * \Psi
$$

We can now make an ansatz for nonperturbative solutions

$$
\Psi=\Psi_{m} \otimes \Psi_{g},
$$

where $\Psi_{g}$ and $\Psi_{m}$ depend purely on ghost and matter degrees of freedom, respectively. Then, since $\mathscr{Q}$ depends only on the ghost modes, eq.(5.3) splits into

$$
\begin{aligned}
\mathscr{Q} \Psi_{g} & =-\Psi_{g} *_{g} \Psi_{g}, \\
\Psi_{m} & =\Psi_{m} *_{m} \Psi_{m},
\end{aligned}
$$

where $*_{g}$ and $*_{m}$ refers to the star product involving only the ghost and matter part. The action for this type of solution becomes

$$
\mathscr{S}(\Psi)=-\frac{1}{6 g_{0}^{2}}\left\langle\Psi_{g}|\mathscr{Q}| \Psi_{g}\right\rangle\left\langle\Psi_{m} \mid \Psi_{m}\right\rangle,
$$

$\left\langle\Psi_{m} \mid \Psi_{m}\right\rangle$ is the ordinary inner product, $\left\langle\Psi_{m}\right|$ being the bpz conjugate of $\left|\Psi_{m}\right\rangle$.

The remarkable characteristic of VSFT is factorization of the matter and ghost part. The solution for the ghost part has been found, (Hata and Kawano 2001), but it does not really matter here since it is universal, and, due to factorization, it drops out of the interesting results. So let us concentrate on the matter part, eq.(5.6). The solutions are projectors of the $*_{m}$ algebra. The $*_{m}$ product is defined as follows

$$
{ }_{123}\left\langle V_{3} \mid \Psi_{1}\right\rangle_{1}\left|\Psi_{2}\right\rangle_{2}={ }_{3}\left\langle\Psi_{1} *_{m} \Psi_{2}\right|
$$

\footnotetext{
${ }^{1}$ It is fair to say that it has never been clarified in what precise sense VSFT is an approximation to SFT.
} 
where the three strings vertex $V_{3}^{(m)}$ is defined in Appendix, (8.2).

The solutions to eq.(5.6) are projectors of the $*_{m}$ algebra. The simplest one is the sliver, (Rastelli et al. 200). Let us recall the main points concerning the sliver solution. It is translationally invariant. As a consequence all momenta can be set to zero. The integration over the momenta can be dropped and the only surviving part in $E$ will be the one involving $V_{n m}^{a b}$, with $n, m \geq 1$. The sliver is defined by

$$
|\Xi\rangle=\mathscr{N} e^{-\frac{1}{2} a^{\dagger} S a^{\dagger}}|0\rangle, \quad a^{\dagger} S a^{\dagger}=\sum_{n, m=1}^{\infty} a_{n}^{\mu \dagger} S_{n m} a_{m}^{v^{\dagger}} \eta_{\mu \nu} .
$$

This state satisfies eq.(5.6) provided the matrix $S$ satisfies the equation

$$
S=V^{11}+\left(V^{12}, V^{21}\right)(1-\Sigma \mathscr{V})^{-1} \Sigma\left(\begin{array}{l}
V^{21} \\
V^{12}
\end{array}\right),
$$

where

$$
\Sigma=\left(\begin{array}{ll}
S & 0 \\
0 & S
\end{array}\right), \quad \mathscr{V}=\left(\begin{array}{ll}
V^{11} & V^{12} \\
V^{21} & V^{22}
\end{array}\right) .
$$

(see the Appendix below for notation). The proof of this fact is well-known, (Kostelecky and Potting 2001). First one expresses eq.(5.11) in terms of the twisted matrices $X=C V^{11}, X_{+}=C V^{12}$ and $X_{-}=C V^{21}$, together with $T=C S=S C$, where $C_{n m}=(-1)^{n} \delta_{n m}$ is the twist matrix. The matrices $X, X_{+}, X_{-}$are mutually commuting, due to eq.(8.7). Then, requiring $T$ to commute with them as well, one can show that eq.(5.11) reduces to the algebraic equation

$$
(1-T)\left(X T^{2}-(1+X) T+X\right)=0 .
$$

Apart form the identity solution, the significant solution is the sliver

$$
T=\frac{1}{2 X}(1+X-\sqrt{(1+3 X)(1-X)})
$$

which evidently commutes with $X, X_{+}, X_{-}$.

The normalization constant $\mathscr{N}$ is calculated to be

$$
\mathscr{N}=(\operatorname{det}(1-\Sigma \mathscr{V}))^{\frac{D}{2}}
$$

where $D=26$. The contribution of the sliver to the matter part of the action (see (5.7)) is given by

$$
\langle\Xi \mid \Xi\rangle=\frac{\mathscr{N}^{2}}{\left(\operatorname{det}\left(1-S^{2}\right)\right)^{\frac{D}{2}}} .
$$

Both eq.(5.14) and (5.15) are ill-defined and need to be regularized.

The sliver solution represents the space-filling D25-brane. In order to find D-brane solutions of lower dimensions we have to define transverse directions, i.e. directions along which the solutions are not translational invariant. The lump solutions are engineered to represent a lower dimensional brane, therefore they are characterized by the breaking of translational invariance along 
a subset of directions. Accordingly we split the three strings vertex into the tensor product of the perpendicular part and the parallel part

$$
\left|V_{3}\right\rangle=\left|V_{3, \perp}\right\rangle \otimes\left|V_{3, \|}\right\rangle
$$

and the exponent $E$, accordingly, as $E=E_{\|}+E_{\perp}$. The parallel part is the same as in the sliver case while the perpendicular part is modified as follows. Following (Rastelli et al. 2002), we denote by $x^{\alpha}, p^{\alpha}, \alpha=1, \ldots, k$ the coordinates and momenta in the transverse directions and introduce the zero mode combinations

$$
a_{0}^{(r) \alpha}=\frac{1}{2} \sqrt{b} \hat{p}^{(r) \alpha}-i \frac{1}{\sqrt{b}} \hat{x}^{(r) \alpha}, \quad a_{0}^{(r) \alpha \dagger}=\frac{1}{2} \sqrt{b} \hat{p}^{(r) \alpha}+i \frac{1}{\sqrt{b}} \hat{x}^{(r) \alpha},
$$

where $\hat{p}^{(r) \alpha}, \hat{x}^{(r) \alpha}$ are the zero momentum and position operator of the $r$-th string, and we have introduced a numerical parameter $b$. It follows that

$$
\left[a_{0}^{(r) \alpha}, a_{0}^{(s) \beta \dagger}\right]=\eta^{\alpha \beta} \delta^{r s} .
$$

Denoting by $\left|\Omega_{b}\right\rangle$ the oscillator vacuum $\left(a_{0}^{\alpha}\left|\Omega_{b}\right\rangle=0\right)$, the relation between the momentum basis and the oscillator basis is defined by

$$
\left|\left\{p^{\alpha}\right\}\right\rangle_{123}=\left(\frac{b}{2 \pi}\right)^{\frac{3}{2}} \exp \left[\sum_{r=1}^{3} \eta^{\alpha \beta}\left(-\frac{b}{4} p_{\alpha}^{(r)} p_{\beta}^{(r)}+\sqrt{b} a_{0 \alpha}^{(r) \dagger} p_{\beta}^{(r)}-\frac{1}{2} a_{0 \alpha}^{(r) \dagger} a_{0 \beta}^{(r) \dagger}\right)\right]\left|\Omega_{b}\right\rangle .
$$

Next we insert this equation inside $E_{\perp}^{\prime}$ and eliminate the momenta along the perpendicular directions by integrating them out. The overall result of this operation is that, while $\left|V_{3, \|}\right\rangle$ is the same as in the ordinary case, we have

$$
\left|V_{3, \perp}\right\rangle^{\prime}=K e^{-E^{\prime}}\left|\Omega_{b}\right\rangle
$$

with

$$
K=\frac{\sqrt{2 \pi b^{3}}}{3\left(V_{00}+b / 2\right)^{2}}, \quad E^{\prime}=\frac{1}{2} \sum_{r, s=1}^{3} \sum_{M, N \geq 0} a_{M}^{(r) \alpha \dagger} V_{M N}^{\prime r s} a_{N}^{(s) \beta \dagger} \eta_{\alpha \beta}
$$

The coefficients $V_{M N}^{\prime r s}$ are given in (Rastelli et al. 2002). The new Neumann coefficients matrices $V^{\prime r s}$ satisfy the same relations as the $V^{r s}$ ones. In particular one can introduce the matrices $X^{\prime} r s=$ $C V^{\prime} r s$, where $C_{N M}=\delta_{N M}$, which turn out to commute with one another. All the relations valid for $X, X_{ \pm}$hold with primed quantities as well. We can therefore repeat verbatim the derivation of the sliver from eq.(5.9) through eq.(5.15). The new solution will have the form (5.9) with $S$ along the parallel directions and $S$ replaced by $S^{\prime}$ along the perpendicular ones. In turn $S^{\prime}$ is obtained as a solution to eq.(5.10) where all the quantities are replaced by primed ones. This amounts to solving eq.(5.12) with primed quantities. Therefore in the transverse directions $S$ is replaced by $S^{\prime}$, given by

$$
S^{\prime}=C T^{\prime}, \quad T^{\prime}=\frac{1}{2 X^{\prime}}\left(1+X^{\prime}-\sqrt{\left(1+3 X^{\prime}\right)\left(1-X^{\prime}\right)}\right) .
$$


In a similar way we have to adapt the normalization and energy formulas $(5.14,5.15)$. Once this is done, one can compute the energy density, which, for a static solution, corresponds to the negative of the action calculated via (5.7) divided by the volume. The absolute value of this energy is not well defined (see below), but one can at least compute the ratio for the tensions of two lumps of contiguous dimensions,

$$
\frac{\tau_{24-k}}{2 \pi \tau_{25-k}}=\frac{3}{\sqrt{2} \pi b^{3}}\left(V_{00}+\frac{b}{2}\right)^{2}\left(\frac{\operatorname{det}\left(1-X^{\prime}\right)^{3} \operatorname{det}\left(1+3 X^{\prime}\right)}{\operatorname{det}(1-X)^{3} \operatorname{det}(1+3 X)}\right)^{\frac{1}{4}} .
$$

This ratio has been proven both numerically and analytically to be 1 . In this way we find the expected value of the ratio of tension of D-branes (in $\alpha^{\prime}=1$ units). Another confirmation of the D-brane interpretation of a lump comes from the space profile, which can be calculated by contracting the lump solution with the coordinate eigenstate $\left|x^{\alpha}\right\rangle$ along the transverse directions. After regularization or after introduction of a constant background $B$ field this profile turns out to be a Gaussian centered at the transverse coordinate origin and thus represents a space-localized solution.

It has been shown that many other solutions exist, similar both to the sliver (for instance the butterfly) and to the lower dimensional lumps. They are all star algebra projectors. In fact it is possible to construct star algebras of such projectors and introduce the notion of orthonormality among them, see (Bonora et al. 2003).

In conclusion, the third conjecture by Sen has more than some ground, although it has not been possible so far to prove it with the same rigour as the first two conjectures. From the lump construction in this section it is evident that one can hardly avoid the oscillator formalism if one wants to find the same kind of solutions in the full SFT. One cannot hope for a factorization of matter and ghosts either. The way is much tougher and passes through a redefinition of the ghost three strings vertex introduced in Appendix, the new vertex being defined with respect to the ghost vacuum $|0\rangle$, rather than to the vacuum $c_{1}|0\rangle$ used in the Appendix. This result has already been achieved (Bonora et al. 2009) and we would now like to briefly review it.

\section{The midpoint ghost vertex}

If one wants to construct a ghost vertex with respect to the ghost vacuum $|0\rangle$ the most natural thing is to choose the natural normal ordering, instead of the conventional one used in Appendix (which is appropriate when the chosen vacuum is $c_{1}|0\rangle$ ). The former is defined by taking $c_{n}$ with $n \leq 1$ and $b_{n}$ with $n \leq-2$ as creation operators. One of the main problems one has to face is the ghosts insertion, which is a priori free. We solve the problem by means of the operator $Y(z)=$ $\frac{1}{2} \partial^{2} c(z) \partial c(z) c(z)$. We insert it at the string midpoint $z=i$ and at its image $-i$. In this way we obtain two vertices, which are defined in the following way.

We define the state $|\hat{0}\rangle=c_{-1} c_{0} c_{1}|0\rangle$ and the tensor product of states

$$
{ }_{123}\langle\hat{\omega}|={ }_{1}\left\langle\hat { 0 } | _ { 2 } \left\langle\left.\hat{0}\right|_{3}\langle\hat{0}|\right.\right.
$$

carrying total $g h=9$. The ghost three strings vertices are

$$
\left\langle\hat{V}_{( \pm i) 3}\right|={ }_{123}\langle\hat{\boldsymbol{\omega}}| e^{\hat{E}_{( \pm i)},} \quad \hat{E}_{( \pm i)}=-\sum_{r, s=1}^{2} \sum_{n, m}^{\infty} c_{n}^{(r)} \hat{V}_{( \pm i) n m}^{r s} b_{m}^{(s)}
$$


where

$$
\hat{V}_{(i) n m}^{r s}=\oint \frac{d z}{2 \pi i} \oint \frac{d w}{2 \pi i} \frac{1}{z^{n-1}} \frac{1}{w^{m+2}}\left(\frac{\left(\frac{d}{d z} f_{r}(z)\right)^{2}}{\frac{d}{d w} f_{s}(w)} \frac{1}{f_{r}(z)-f_{s}(w)}\left(\frac{f_{s}(w)}{f_{r}(z)}\right)^{3}-\frac{\delta^{r s}}{z-w}\right)
$$

and

$$
\hat{V}_{(-i) n m}^{r s}=\oint \frac{d z}{2 \pi i} \oint \frac{d w}{2 \pi i} \frac{1}{z^{n-1}} \frac{1}{w^{m+2}}\left(\frac{\left(\frac{d}{d z} f_{r}(z)\right)^{2}}{\frac{d}{d w} f_{s}(w)} \frac{1}{f_{r}(z)-f_{s}(w)}-\frac{\delta^{r s}}{z-w}\right)
$$

The first three rows of these matrices vanish, they are complex and satisfy the twist covariance property

$$
\hat{V}_{(i) n m}^{r s}=(-1)^{n+m} \hat{V}_{(-i) n m}^{s r}
$$

that is the vertex with $Y$ insertion at $i$ is twist conjugate to the one with insertion at $-i$.

Using the twist matrix $C_{n m}=(-1)^{n} \delta_{n m}$ one can define the Neumann matrices $X_{( \pm i)}^{r s}=C V_{( \pm i)}^{r s}$. With a minor modification one gets the matrices $X_{( \pm i)}^{\prime r s}$ which mutually commute. Defining $X^{\prime}=$ $X^{\prime r r}, X^{\prime+}=X^{\prime 12}$ and $X^{\prime-}=X^{\prime 21}$ one can verify that

$$
\begin{aligned}
& X_{(i)}^{\prime}+X_{(i)}^{\prime+}+X_{(i)}^{\prime-}=1+\mathfrak{E} \\
& X_{(i)}^{\prime+} X_{(i)}^{\prime-}=X_{(i)}^{\prime 2}-X_{(i)}^{\prime}+\mathfrak{E} \\
& X_{(i)}^{\prime 2}+\left(X_{(i)}^{\prime+}\right)^{2}+\left(X_{(i)}^{\prime-}\right)^{2}=1 \\
& \left(X_{(i)}^{\prime+}\right)^{3}+\left(X_{(i)}^{\prime-}\right)^{3}=1+2 X_{(i)}^{\prime 3}-3 X_{(i)}^{\prime 2}-2 \mathfrak{E}
\end{aligned}
$$

Apart from $\mathfrak{E}$ these are the same relations one finds for the matter Neumann matrices. The matrix $\mathfrak{E}$ commutes with all the $X_{(i)}^{\prime} s$ and in fact it represents a minor complications. One can prove moreover that all such matrices are diagonal in the basis in which the matrix $G$ defining $K_{1}=L_{1}+L_{-1}$ is.

Analogous relations can be obtained for the $-i$ inserted vertex by twist conjugation. All these properties allow us to carry out the star product much in the same way as for matter string states. Let us consider the star product of two squeezed states such as

$$
|S\rangle=\mathscr{N} \exp \left(c^{\dagger} S b^{\dagger}\right)|0\rangle
$$

i.e.

$$
\left\langle\hat{V}_{3} \mid S_{1}\right\rangle\left|S_{2}\right\rangle=\left\langle\hat{S}_{12}\right|
$$

The matrix $\hat{S}_{12}=C T_{12}$ is given by the familiar formula

$$
T_{12}=X+\left(X^{+}, X^{-}\right) \frac{1}{1-\Sigma_{12} \mathscr{V}} \Sigma_{12}\left(\begin{array}{l}
X^{-} \\
X^{+}
\end{array}\right)
$$

where

$$
\Sigma_{12}=\left(\begin{array}{cc}
C S_{1} & 0 \\
0 & C S_{2}
\end{array}\right), \quad \mathscr{V}=\left(\begin{array}{cc}
X & X^{+} \\
X^{-} & X
\end{array}\right)
$$


These formulas hold for both vertices with insertion at $i$ and $-i$, respectively. It was shown in (Bonora et al. 09a) that if $\left|S_{1}\right\rangle$ and $\left|S_{2}\right\rangle$ represent wedge states (with ghost number 0, i.e. without ghost insertions) eq.(6.8) can be diagonalized in the $G$ basis and the wedge states Neumann matrices can be shown to satisfy the recursion relation (4.3), thus suggesting that our definition of the three strings vertices is correct. However this is not enough to guarantee that the star product is the correct one. For we remark that the states like (6.6) are defined on the ghost number 0 vacuum $|0\rangle$, while the resulting state in the RHS of (6.7) is defined in the ghost number 3 vacuum $\langle\hat{0}|$. Therefore $\left\langle\hat{S}_{( \pm i) 12}\right|$ is not yet the star product of $\left|S_{1}\right\rangle$ and $\left|S_{2}\right\rangle$. To certify this one must be able to reconstruct the ghost number number 0 state corresponding to the ghost number 3 ones $\left\langle\hat{S}_{( \pm i) 12}\right|$. The explicit reconstruction goes far beyond the scope of this short review, but it has actually been done in (Bonora et al. 2009b). It has been shown there that by using both states $\left\langle\hat{S}_{(i) 12}\right|$ and $\left\langle\hat{S}_{(-i) 12}\right|$ one can indeed reconstruct the expected ghost number 0 wedge state, thus closing the circle. This shows that there exists a definite procedure to perform the star product of wedge (and other) states using (only) the oscillator formalism.

Now, Y.Okawa (Okawa 06) has shown that, if we are allowed to star-multiply the wedge states as in (4.3), if $|1\rangle$ is the identity state and if the following properties hold

$$
\begin{aligned}
& Q\left(\phi_{1} \star \phi_{2}\right)=\left(Q \psi_{1}\right) \star \psi_{2}+(-1)^{\left|\phi_{1}\right|} \phi_{1} \star\left(Q \phi_{2}\right) \\
& Q^{2}=0 \\
& Q|0\rangle=0 \\
& Q c_{1}|0\rangle=-c_{0} c_{1}|0\rangle \\
& \left(B_{1}^{L}\right)^{2}=\left(B_{1}^{R}\right)^{2}=0 \\
& \left(B_{1}^{R}+B_{1}^{L}\right)|0\rangle=0 \\
& \left(B_{1}^{R}+B_{1}^{L}\right) c_{1}|0\rangle=|0\rangle \\
& \left\{Q, B_{1}^{L}\right\}=K_{1}^{L} \\
& \left\{Q, B_{1}^{R}\right\}=K_{1}^{R} \\
& \left(B_{1}^{R} \phi_{1}\right) \star \phi_{2}=-(-1)^{\left|\phi_{1}\right|} \phi_{1} \star\left(B_{1}^{L} \phi_{2}\right)
\end{aligned}
$$

where $\phi_{1}$ and $\phi_{2}$ are any two string states, it is a simple matter of algebra to prove that $\Psi_{\lambda}=$ $\sum_{n=0}^{\infty} \lambda^{n+1} \psi_{n}^{\prime}$ is a solution of the SFT equation of motion.

The relations (6.9), except the first and the last, are elementary to prove in the oscillator formalism. Assuming, for simplicity, the validity of the first and last relation, we can conclude that Schnabl's solution can be demonstrated also in the oscillator formalism. We hope this fact may open the way to the analytic proof of third Sen's conjecture.

\section{Open-closed string duality}

Sen's conjectures tell us that the (locally) stable SFT vacuum is in fact the closed string vacuum. Apart from the formal proof of Ellwood and Schnabl, there are independent arguments. From a physical point of view the D-branes in question are unstable and it has been shown (Lambert et al. 2003, Sen 2003a, Sen 2003b, Gaiotto et al. 2004) that such branes decay into heavy closed string modes with negligible transverse velocity. From a formal point of view we expect that, since 
the theory simply changes vacuum, the closed string degrees of freedom may be expressible in terms of the old ones. In the last part of this presentation, I would like to give some indication that perhaps this is the case (for the following construction, see (Are'feva et al. 2002, Bonora et al. 2006)).

Using the sliver coefficients matrix $S_{n m}$ let us define the operators

$$
s^{\mu}=\omega\left(a^{\mu}+S a^{\mu \dagger}\right)=\left(a^{\mu}+S a^{\mu \dagger}\right) \omega, \quad \omega=\frac{1}{\sqrt{1-S^{2}}}
$$

and the conjugate ones, where the labels $n, m$ running from 1 to $+\infty$ are understood ( $S a$ means $\sum_{m=1}^{\infty} S_{n m} a_{m}$, etc.). Using the algebra of open string creation and annihilation operators, these new operators can be shown to satisfy

$$
\left[s_{m}^{\mu}, s_{n}^{v^{\dagger}}\right]=\delta_{n m} \eta^{\mu v}
$$

Moreover, understanding the Lorentz indices,

$$
s_{n}|\Xi\rangle=\mathscr{N} e^{-\frac{1}{2} a^{\dagger} S a^{\dagger}} \omega\left(a-S a^{\dagger}+S a^{\dagger}\right)|0\rangle=0
$$

Therefore the combinations $s_{n}$ represent Bogoliubov transformations of the original oscillators, which map the Fock space based on the initial vacuum $|0\rangle$ to a new Fock space in which the role of vacuum is played by the sliver.

One can define (Bonora et al. 2006) coefficients $b_{n l}$ and $\tilde{b}_{n l}$, so that, setting,

$$
\beta_{m}^{\mu}=\sum_{l=1}^{\infty} b_{m l} s_{l}^{\mu}, \quad \tilde{\beta}_{m}^{\mu}=-\sum_{l=1}^{\infty} \tilde{b}_{m l} s_{l}^{\mu}
$$

these operators satisfy the algebra

$$
\begin{aligned}
& {\left[\beta_{m}^{\mu}, \beta_{n}^{v^{\dagger}}\right]=\delta_{m, n} \eta^{\mu v}} \\
& {\left[\tilde{\beta}_{m}^{\mu}, \tilde{\beta}_{n}^{v^{\dagger}}\right]=\delta_{m, n} \eta^{\mu v}}
\end{aligned}
$$

while all the other commutators vanish.

The operators $\beta_{n}$ and $\tilde{\beta}_{n}$ and their conjugates are characterized by a Heisenberg algebra isomorphic to the algebra of closed string creation and annihilation operators. They are natural candidates as closed string creation and annihilation operators. For the same reason it is natural to interpret the sliver $|\Xi\rangle$ as the closed string vacuum $\left|0_{c}\right\rangle$.

This is very straightforward, but it takes a long way before we are able to claim that they do represent the closed string oscillators and vacuum, respectively. Let us start first by considering a complete set of states as possible candidates of perturbative closed string states. To this end we define sequences of natural numbers $\mathbf{n}=n_{1}, n_{2}, \ldots$, where the label $l$ in $n_{l}$ corresponds to the oscillator type. For every type $l$ half string oscillator we will have a collection of symmetric Lorentz indices $\mu_{1}^{l}, \mu_{2}^{l}, \ldots, \mu_{n_{l}}^{l}$. Then for any two sequences $\mathbf{n}$ and $\mathbf{m}$ we define the states:

$$
\Lambda^{\left\{\mu_{1} \ldots \mu_{\mathbf{n}}\right\},\left\{v_{1} \ldots v_{\mathbf{m}}\right\}}=\prod_{l, r=1}^{\infty} \frac{(-1)^{m_{r}}}{\sqrt{n_{l} ! m_{r} !}} \beta_{l}^{\mu_{1}^{l} \dagger} \ldots \beta_{l}^{\mu_{n_{l}}^{l} \dagger} \tilde{\beta}_{r}^{v_{1}^{r} \dagger} \ldots \tilde{\beta}_{r}^{v_{m_{r}}^{r} \dagger}|\Xi\rangle
$$


Note that in this new representation the labels $(n, m)$ are naturally interpreted as two independent (left/right) spin quantities (number of symmetric indices).

The states (7.5) are string fields in the original OSFT and look like perturbative closed string states in the new vacuum. The relevant question is now: what are the (open) string fields that correspond to closed string Fock states created under the above correspondence? By closed string states we mean both off-shell and on-shell states. For instance a graviton state with momentum $k$ in closed string theory is given by

$$
\theta_{\mu \nu} \alpha_{1}^{\mu \dagger} \alpha_{1}^{v \dagger}\left|0_{c}, k\right\rangle
$$

where $\left|0_{c}, k\right\rangle$ is the closed string vacuum with momentum $k$, and the symmetric tensor $\theta_{\mu \nu}$ is the polarization. This state is on-shell when $k^{2}=0$ and $\theta_{\mu v} k^{v}=0$. When the latter conditions are not satisfied the graviton is off-shell. Off-shell states are not so generic as one might think, they must satisfy precise conditions: they must have definite momentum (i.e. the holomorphic and antiholomorphic momenta must be equal) and they must be level-matched. In the following we will deal with off-shell closed string states and we will focus, for the sake of simplicity, only on zero momentum states.

It is evident from the above that there is a correspondence between (zero momentum) states in the Fock space of the closed string theory and open string fields of the type (7.5). The question is: what are the string fields that correspond to off-shell states in the closed string theory?

To start with we define the level matching condition by means of

$$
N_{L}=\sum_{n=1}^{\infty} n \beta_{n}^{\dagger} \cdot \beta_{n}, \quad N_{R}=\sum_{n=1}^{\infty} n \tilde{\beta}_{n}^{\dagger} \cdot \tilde{\beta}_{n},
$$

Off-shell states are characterized in particular by the condition $N_{R}=N_{L}=N$, where the number $N$ specifies the level of the state. They are in general combination of monomials of $\beta$ and $\tilde{\beta}$ applied to the vacuum with arbitrary coefficients. Now one can prove the following statement:

Closed string Fock space states of given level, satisfying the level matching condition, can always be decomposed into combinations of states of the type (7.5) that are *-algebra projectors. Loosely speaking, level-matched states of the closed string Fock space come from star algebra projectors of the OSFT.

The proof can be found in (Bonora et al., 2006), where it is also explained how to modify these states by assigning an appropriate momentum. All this is still rather formal. However one can put forward a more compelling argument.

One can prove the identity

$$
\sum_{n} \beta_{n}^{\mu \dagger} \tilde{\beta}_{n}^{v \dagger} \eta_{\mu v}=\frac{1}{2} \sum_{k=1}^{\infty} s_{k}^{\mu \dagger} C_{k l} s_{l}^{\nu^{\dagger}} \eta_{\mu \nu}
$$

from which it follows that

$$
e^{-\sum_{n} \beta_{n}^{\mu \dagger} \tilde{\beta}_{n}^{v \dagger} \eta_{\mu v}}\left|0_{c}\right\rangle=e^{-\frac{1}{2} \sum_{k=1}^{\infty} s_{k}^{\mu \dagger} C_{k l} s_{l}^{v \dagger} \eta_{\mu v}}|\Xi\rangle \sim e^{-\frac{1}{2} \sum_{k=1}^{\infty} a_{k}^{\mu \dagger} C_{k l} a_{l}^{v \dagger} \eta_{\mu v}}|0\rangle
$$

where $|0\rangle$ is the original open string vacuum. The LHS has the form of a boundary state in closed string theory, representing a D-brane filling all the space (there are no transverse directions). Suppose we wish to represent instead a Dk-brane (with $25-k$ transverse directions and $k+1$ parallel 
ones, including time). Then the oscillator part of the corresponding boundary state in closed string theory is the tensor product of a factor like the LHS of eq.(7.8) and a transverse factor. This transverse factor breaks translational invariance and, consequently, it is natural to assume it takes the form of a lump. The construction is again given in (Bonora et al., 2006). Here we report the results. Denoting with a prime the new creation operators $s_{n} \rightarrow s_{n}^{\prime}$ we find the analog of (7.8) for the transverse directions:

$$
e^{\sum_{n} \beta_{n}^{i \dagger} \tilde{\beta}_{n}^{j \dagger} \eta_{i j}}\left|0_{c}\right\rangle=e^{-\frac{1}{2} \sum_{k=1}^{\infty} s_{k}^{i \dagger} C_{k l} s_{l}^{\prime j \dagger} \eta_{i j}}|\Xi\rangle \sim e^{-\frac{1}{2} \sum_{k=1}^{\infty} a_{k}^{i \dagger} C_{k l} a_{l}^{j \dagger} \eta_{i j}}|0\rangle
$$

where again $|0\rangle$ is the original open string vacuum.

As one can see, while the exponents of the LHS's of these two equations have opposite sign, the RHS of the two equations takes the same form. This miracle has to be traced back to the twist properties of the 'sliver basis' and the 'lump basis' and it is certainly not accidental.

Now taking the tensor product of (7.8) and (7.9), the resulting state in the LHS is proportional to the boundary state in closed string theory, while the right hand side is the identity state in open string field theory. The boundary state represents a Dk-brane in the closed string language. The identity state represents absence of interaction in the open string field theory language. We can interpret the above equality in the following way: closed strings are reflected by the Dk-brane (they feel it). Open strings live on the Dk-brane, therefore they perceive the corresponding state as an identity state (they do not feel it).

Even after this positive check there is still much to be done in order to represent closed strings in terms of open string degrees of freedom. Perhaps the approach outlined in this section is still too naive. But, at least, it shows that the solution to this problem may be within our reach.

\section{Appendix: the three strings vertex}

The role of the three strings interaction in SFT is so crucial that, notwithstanding the elegance and simplicity of the CFT formulation, we are lucky that another powerful alternative method exists, which becomes very handy in many circumstances. This is based on the oscillator formalism and utilizes the so-called three strings vertex. Indeed, as was anticipated above we can represent the star product of two string fields $\Psi_{1}$ and $\Psi_{2}$ in the following way

$$
\left\langle V_{3}|| \Psi_{1}\right\rangle\left|\Psi_{2}\right\rangle=\left\langle\Psi_{1} \star \Psi_{2}\right\rangle
$$

We split the $V_{3}$ vertex into matter and ghost part, $V_{3}=V_{3}^{(m)} \otimes V_{3}^{(g h)}$. Let us start with the matter part. The matter vertex $V_{3}^{(m)}$ is given by

$$
\left|V_{3}^{(m)}\right\rangle=\int d^{26} p_{(1)} d^{26} p_{(2)} d^{26} p_{(3)} \delta^{26}\left(p_{(1)}+p_{(2)}+p_{(3)}\right) \exp (-E)|0, p\rangle_{123},
$$

where

$$
E=\sum_{a, b=1}^{3}\left(\frac{1}{2} \sum_{m, n \geq 1} \eta_{\mu v} a_{m}^{(a) \mu \dagger} V_{m n}^{a b} a_{n}^{(b) v \dagger}+\sum_{n \geq 1} \eta_{\mu v} p_{(a)}^{\mu} V_{0 n}^{a b} a_{n}^{(b) v \dagger}+\frac{1}{2} \eta_{\mu v} p_{(a)}^{\mu} V_{00}^{a b} p_{(b)}^{v}\right)
$$


Summation over the Lorentz indices $\mu, v=0, \ldots, 25$ is understood. The operators $a_{m}^{(a) \mu}, a_{m}^{(a) \mu \dagger}$ denote the non-zero modes matter oscillators of the $a$-th string (they are related to the previously introduced $\alpha$ oscillators by $m a_{m}=\alpha_{m}$ ), which satisfy

$$
\left[a_{m}^{(a) \mu}, a_{n}^{(b) v^{\dagger}}\right]=\eta^{\mu v} \delta_{m n} \delta^{a b}, \quad m, n \geq 1,
$$

$p_{(a)}$ is the momentum of the $a$-th string and $|0, p\rangle_{123} \equiv\left|p_{(1)}\right\rangle \otimes\left|p_{(2)}\right\rangle \otimes\left|p_{(3)}\right\rangle$ is the tensor product of the Fock vacuum states relative to the three strings. $\left|p_{(a)}\right\rangle$ is annihilated by the annihilation operators $a_{m}^{(a) \mu}$ and it is eigenstate of the momentum operator $\hat{p}_{(a)}^{\mu}$ with eigenvalue $p_{(a)}^{\mu}$. The normalization is

$$
\left\langle p_{(a)} \mid p_{(b)}^{\prime}\right\rangle=\delta_{a b} \delta^{26}\left(p+p^{\prime}\right) .
$$

In order to get $\left\langle V_{3}\right|$ one has to use the $b p z$ conjugation properties of the oscillators

$$
\operatorname{bpz}\left(a_{n}^{(a) \mu}\right)=(-1)^{n+1} a_{-n}^{(a) \mu} .
$$

$\left\langle V_{3}\right|$ is the $b p z$ conjugate of $\left|V_{3}\right\rangle$ (the $b p z$ conjugation does not alter the order of the oscillators). In eq.(8.1) the LHS represent the contraction of two bra's with two ket's. The result is a bra from which by $b p z$ conjugation one obtains $\left|\Psi_{1} \star \Psi_{2}\right\rangle$.

The coefficients $V_{n m}^{a b}$ contain all the information about the star product and one needs to know their explicit expression. To this end we compute the Neumann coefficients $N_{n m}^{a b}$, which are related to them in a simple way. For any three string fields we require that

$$
\left\langle f_{1} \circ \Psi_{1}(0) f_{2} \circ \Psi_{2}(0) f_{3} \circ \Psi_{3}(0)=\left\langle V_{123} \mid \Psi_{1}\right\rangle_{1} \mid \Psi_{2}\right\rangle_{2}\left|\Psi_{3}\right\rangle_{3}
$$

A simple way to exploit this is to consider the string propagator at two generic points of the disk (see above). The Neumann coefficients $N_{N M}^{a b}$ are nothing but the Fourier modes of the propagator with respect to the original coordinates $z_{a}$.

Here, for simplicity, we only deal with the Neumann coefficients not involving the zero mode $p_{\mu}^{(a)}$. The Neumann coefficients $N_{m n}^{a b}$ with $n, m>0$ are given by, (Leclair et al. 1989),

$$
\begin{aligned}
N_{m n}^{a b} & =\left\langle V_{123}\left|\alpha_{-n}^{(a)} \alpha_{-m}^{(b)}\right| 0\right\rangle_{123} \\
& =-\frac{1}{n m} \oint \frac{d z}{2 \pi i} \oint \frac{d w}{2 \pi i} \frac{1}{z^{n}} \frac{1}{w^{m}} f_{a}^{\prime}(z) \frac{1}{\left(f_{a}(z)-f_{b}(w)\right)^{2}} f_{b}^{\prime}(w),
\end{aligned}
$$

where the contour integrals are understood around the origin. It is easy to check that

$$
\begin{aligned}
& N_{m n}^{a b}=N_{n m}^{b a}, \\
& N_{m n}^{a b}=(-1)^{n+m} N_{m n}^{b a}, \\
& N_{m n}^{a b}=N_{m n}^{a+1, b+1} .
\end{aligned}
$$

In the last equation the upper indices are defined mod 3.

We will not do it here, but it is easy to make the identification

$$
V_{n m}^{a b}=(-1)^{n+m} \sqrt{n m} N_{n m}^{a b},
$$


and to establish the fundamental commutativity relation (written in matrix notation)

$$
\left[C V^{a b}, C V^{a^{\prime} b^{\prime}}\right]=0
$$

for any $a, b, a^{\prime}, b^{\prime}$, where $C$ is the twist matrix $C_{n m}=(-1)^{n} \delta_{n, m}$. Similar commutativity relations can be obtained also for the coefficient matrices involving the zero mode $p_{\mu}$.

Next, let us consider the ghost vertex. To start with we define, in the ghost sector, the vacuum states $|\hat{0}\rangle$ and $|\dot{0}\rangle$ as follows

$$
|\hat{0}\rangle=c_{0} c_{1}|0\rangle, \quad|\dot{0}\rangle=c_{1}|0\rangle,
$$

where $|0\rangle$ is the usual $S L(2, R)$ invariant vacuum. Using $b p z$ conjugation

$$
c_{n} \rightarrow(-1)^{n+1} c_{-n}, \quad b_{n} \rightarrow(-1)^{n-2} b_{-n}, \quad|0\rangle \rightarrow\langle 0|,
$$

one can define conjugate states. It is important that, when applied to products of oscillators, the $b p z$ conjugation does not change the order of the factors.

The three strings interaction vertex is defined again as a squeezed operator acting on three copies of the $b c$ Fock space

$$
\left\langle\tilde{V}_{3}\right|={ }_{1}\langle\hat{0}|{ }_{2}\langle\hat{0}|{ }_{3}\langle\hat{0}| e^{\tilde{E}}, \quad \tilde{E}=\sum_{a, b=1}^{3} \sum_{n, m}^{\infty} c_{n}^{(a)} \tilde{V}_{n m}^{a b} b_{m}^{(b)} .
$$

The Neumann coefficients $\tilde{V}_{n m}^{a b}$ are given by the contraction of the $b c$ oscillators on the unit disk. They represent Fourier components of the $S L(2, R)$ invariant $b c$ propagator (i.e. the propagator in which the zero modes have been inserted at fixed points $\left.\zeta_{i}, i=1,2,3\right)$ :

$$
\langle b(z) c(w)\rangle=\frac{1}{z-w} \prod_{i=1}^{3} \frac{w-\zeta_{i}}{z-\zeta_{i}} .
$$

Taking into account the conformal properties of the $b, c$ fields and inserting the zero modes at zero $\zeta_{i}=0$, we get

$$
\begin{aligned}
\tilde{V}_{n m}^{a b} & =\left\langle\tilde{V}_{123}\left|b_{-n}^{(a)} c_{-m}^{(b)}\right| \dot{0}\right\rangle_{123} \\
& =\oint \frac{d z}{2 \pi i} \oint \frac{d w}{2 \pi i} \frac{1}{z^{n-1}} \frac{1}{w^{m+2}}\left(f_{a}^{\prime}(z)\right)^{2} \frac{-1}{f_{a}(z)-f_{b}(w)} \frac{f^{3}(w)-1}{f^{3}(z)-1}\left(f_{b}^{\prime}(w)\right)^{-1} .
\end{aligned}
$$

It is straightforward to check that

$$
\tilde{V}_{n m}^{a b}=\tilde{V}_{n m}^{a+1, b+1}
$$

and

$$
\tilde{V}_{n m}^{a b}=(-1)^{n+m} \tilde{V}_{n m}^{b a}
$$

Moreover, it is possible to prove that, see for instance (Bonora et al. 2003),

$$
\left[\tilde{X}^{a b}, \tilde{X}^{a^{\prime} b^{\prime}}\right]=0
$$

where, once again, $\tilde{X}^{a b}=C \tilde{V}^{a b}$. 


\section{References}

[1] E.Witten, Noncommutative Geometry and String Field Theory, Nucl.Phys. B268 (1986) 253.

[2] A.Sen Tachyon Condensation on the Brane Antibrane System JHEP 9808 (1998) 012, [hep-th/9805170]. BPS D-branes on Non-supersymmetric Cycles, JHEP 9812 (1998) 021, [hep-th/9812031].

[3] A.Sen Descent Relations among Bosonic D-Branes, Int.J.Mod.Phys. A14 (1999) 4061, [hep-th/9902105].

[4] A. Sen, Rolling tachyon, JHEP 0204 (2002) 048 [arXiv:hep-th/0203211].

[5] A. Sen, Open-closed duality: Lessons from matrix model, Mod. Phys. Lett. A 19 (2004) 841 [arXiv:hep-th/0308068].

[6] A. Sen, Open and closed strings from unstable D-branes, Phys. Rev. D 68 (2003) 106003, [arXiv:hep-th/0305011].

[7] M. Schnabl, Analytic solution for tachyon condensation in open string field theory, Adv. Theor. Math. Phys. 10 (2006) 433 [arXiv:hep-th/0511286].

[8] I. Ellwood and M. Schnabl, Proof of vanishing cohomology at the tachyon vacuum, JHEP 0702 (2007) 096 [arXiv:hep-th/0606142].

[9] Y. Okawa, Comments on Schnabl's analytic solution for tachyon condensation in Witten's open string field theory, JHEP 0604 (2006) 055 [arXiv:hep-th/0603159].

[10] W. Taylor and B. Zwiebach, D-branes, tachyons, and string field theory, [hep-th/0311017].

[11] K.Ohmori, A review of tachyon condensation in open string field theories, [hep-th/0102085].

[12] I.Ya.Aref'eva, D.M.Belov, A.A.Giryavets, A.S.Koshelev, P.B.Medvedev, Noncommutative field theories and (super)string field theories, [hep-th/0111208].

[13] L.Bonora, C.Maccaferri, D.Mamone and M.Salizzoni, Topics in string field theory, [hep-th/0304270].

[14] E. Fuchs and M. Kroyter, Analytical Solutions of Open String Field Theory, [hep-th/0807.4722].

[15] A.Leclair, M.E.Peskin, C.R.Preitschopf, String Field Theory on the Conformal Plane. (I) Kinematical Principles, Nucl.Phys. B317 (1989) 411.

[16] L.Rastelli, A.Sen and B.Zwiebach, String field theory around the tachyon vacuum, Adv. Theor. Math. Phys. 5 (2002) 353 [hep-th/0012251].

[17] L.Rastelli, A.Sen and B.Zwiebach, Vacuum string field theory, [hep-th/0106010].

[18] L.Rastelli, A.Sen and B.Zwiebach, Star Algebra Spectroscopy, JHEP 0203 (2002) 029 [hep-th/0111281].

[19] H.Hata and T.Kawano, Open string states around a classical solution in vacuum string field theory, JHEP 0111 (2001) 038 [hep-th/0108150].

[20] V.A.Kostelecky and R.Potting, Analytical construction of a nonperturbative vacuum for the open bosonic string, Phys. Rev. D 63 (2001) 046007 [hep-th/0008252].

[21] N. Lambert, H. Liu and J. Maldacena, “Closed strings from decaying D-branes," arXiv:hep-th/0303139.

[22] D. Gaiotto, N. Itzhaki and L. Rastelli, “Closed strings as imaginary D-branes,” Nucl.Phys.B, 688, 70, [arXiv:hep-th/0304192]. 
[23] L. Bonora, N. Bouatta and C. Maccaferri, Toward open-closed string duality, [hep-th/0609182].

[24] L. Bonora, C. Maccaferri, R. J. Scherer Santos and D. D. Tolla, Ghost story. I. Wedge states in the oscillator formalism, JHEP 0709 (2007) 061 [arXiv:0706.1025 [hep-th]].

[25] L. Bonora, C. Maccaferri, R.J. Scherer Santos and D.D. Tolla, Ghost story. II. The midpoint ghost vertex, [arXiv:0908.0055 [hep-th]].

[26] L. Bonora, C. Maccaferri and D. D. Tolla, Ghost story. III. Back to ghost number zero, [arXiv:0908.0056 [hep-th]]. 